Théologiques

Théologiques

\title{
John Hagee, dirigeant du groupe chrétien sioniste américain, Christians United For Israel
}

\section{Katia Batut-Lucas}

Volume 22, numéro 2, 2014

URI : https://id.erudit.org/iderudit/1035693ar

DOI : https://doi.org/10.7202/1035693ar

Aller au sommaire du numéro

\section{Éditeur(s)}

Faculté de théologie et de sciences des religions, Université de Montréal

ISSN

1188-7109 (imprimé)

1492-1413 (numérique)

Découvrir la revue

Citer cet article

Batut-Lucas, K. (2014). John Hagee, dirigeant du groupe chrétien sioniste américain, Christians United For Israel. Théologiques, 22(2), 229-252.

https://doi.org/10.7202/1035693ar
Résumé de l'article

Cet article porte sur John Hagee et son parcours pour devenir le dirigeant du groupe chrétien sioniste le plus grand et le plus médiatisé aux États-Unis et dans le monde, le Christians United For Israel (CUFI). Depuis 2006, ce pasteur s'est fait connaître du grand public à cause de son soutien inconditionnel à l'État hébreu. Comment Hagee en est-il arrivé à créer le plus grand groupe de pression chrétien sioniste qui existe de nos jours ? Comment est-il devenu l'am d'Israël et ce chef de file si remarqué ? De quel milieu évangélique est-il issu? Cet article veut ainsi comprendre comment un pasteur évangélique est devenu une figure politique médiatisée et comment il a pu rallier bon nombre d'évangéliques à le suivre dans son activisme pro-Israël. Hagee est une figure charismatique. Notre méthodologie se fonde sur des études de terrain, des entretiens et des observations. 


\title{
John Hagee, dirigeant du groupe chrétien sioniste américain, Christians United For Israel
}

\author{
Katia Batut-Lucas* \\ Sciences des religions, \\ Université de Montréal, Canada \\ Université Bordeaux-Montaigne, France
}

Le pasteur John Hagee est le dirigeant évangélique du groupe chrétien sioniste le plus grand et le plus médiatisé aux États-Unis, le Christians United For Israel (CUFI), en français: Chrétiens Unis Pour Israël. À la tête d'un empire religieux à San Antonio au Texas, Hagee dirige une megachurch $^{1}$, Cornerstone Church, de vingt mille membres. Le CUFI représente le théâtre même du sionisme évangélique depuis 2006. Lors des élections présidentielles de 2008, Hagee commença à bien mieux se faire connaître du grand public, et ce internationalement, en soutenant le candidat républicain John McCain, mais aussi par ses discours anti-catholicisme, antiislam et anti-homosexualité. En 2010, il condamna l'Angleterre parce que les autorités britanniques avaient auparavant déclaré que le mur des Lamentations ne pouvait pas être considéré comme un monument touristique puisqu'il était situé en territoires occupés : selon Hagee, les émissions

* Katia Batut-Lucas a obtenu un doctorat conjoint de l'Université de Montréal (sciences des religions) et de l'Université Bordeaux-Montaigne (études anglophones). Sa thèse de doctorat s'intitule "Le sionisme chrétien contemporain aux États-Unis, entre religion et politique »; elle porte sur le rapport religion/politique du groupe de pression évangélique sioniste le plus important aux États-Unis, le Christians United For Israel. Elle a récemment publié (2014) «Le pèlerinage et le sionisme chrétien aux États-Unis. Le cas du Christians United For Israel», dans L. Chantre et al., dir., Politiques du pèlerinage, Rennes, Presses universitaires de Rennes. Pour toute correspondance: katia.batut@umontreal.ca.

1. Sur les "megachurches", voir Fath (2005). Cette Église évangélique est "nondénominationnelle » car elle se caractérise par son indépendance de tout autre réseau ecclésial (par exemple baptistes ou luthériennes, assemblées de Dieu, etc.). 
de vapeur provenant de l'éruption volcanique de l'Islande, qui recouvraient le ciel de la Grande-Bretagne, auraient été le signe qu'elle avait brisé l'alliance de Dieu avec Israël (Van Ingen 2010), amenant la malédiction sur les Britanniques.

Comment ce pasteur en est-il arrivé à créer le plus grand groupe de pression chrétien sioniste? Comment est-il devenu l'ami d'Israël et ce chef de file si remarqué ? Il existe des écrits sur le sionisme chrétien, précisons évangélique (Ariel 2011; Belin 2011; Fath 2005; Sizer 2006; Spector 2009; Weber 2004), mais aucun ne développe suffisamment la vie de Hagee pour comprendre le passage d'une telle figure, du religieux au politique, même s'il est reconnu comme la personnalité la plus importante du mouvement (Belin 2011; Kicarofe 2009). Il y a un manque à combler alors qu'il existe des biographies écrites sur des dirigeants incontournables du christianisme évangélique, tels que Jerry Falwell (Harding 2001) ou Billy Graham (Wacker 2014). Nous ne prétendons pas offrir une biographie complète de Hagee, nous n'en avons pas la place ici. Mais, dans une perspective large, nous souhaitons apporter des matériaux sur ce dirigeant, à quiconque souhaite mieux comprendre son parcours.

Nous nous fondons sur les livres de Hagee, ainsi que sur un recueil d'articles de presse et d'anecdotes sur ce personnage, publié par sa fille ainée Tina et sa femme Diana (Hagee et Ketterling 2008). En 2011 et 2013, nous avons mené des observations et une quarantaine d'entretiens avec des sympathisants du CUFI ${ }^{2}$, pendant lesquels certains parlaient de Hagee, qui a également été interrogé. Les croyants avaient entre 21 et 84 ans. Le plus jeune était étudiant, le plus âgé, retraité. Certains étaient des pasteurs laïques, d'autres enseignants, mères au foyer, hommes d'affaires, vendeurs, travailleurs autonomes; un était artiste, un autre journaliste, etc.

2. Cette recherche qualitative est extraite de notre recherche doctorale. Les questions portaient sur la manière dont ces croyants étaient devenus des «chrétiens sionistes » et sur leur compréhension du sionisme chrétien. La question initiale lors des entretiens dirigés était la suivante: First give us a bit of background about yourself. How did you become a Christian Zionist?), sauf pour Hagee et son porte-parole. La première question posée au pasteur Hagee a été: God revealed to you the importance of supporting Israel. But are there any other Christian people who could help you to become a Christian Zionist leader?. Diverses questions ont été posées, telles que: Could you define a Christian Zionist? /Christian Zionism?, How would you describe Pastor Hagee? Nous ne donnons pas les noms des croyants, à l'exception des dirigeants, pour garder leur anonymat. 
La majorité étaient membre de l'Église de Hagee, quelques-uns n'allaient jamais dans son assemblée ${ }^{3}$.

Nous verrons comment Hagee est devenu le dirigeant du CUFI pour saisir comment ce pasteur peut avoir autant d'influence dans le milieu évangélique. Nous nous pencherons sur l'enfance de Hagee jusqu'à ce qu'il devienne le dirigeant du CUFI. Notre intérêt portera rapidement sur son organisation. Puis, nous verrons en quoi Hagee représente une figure charismatique. Auparavant, nous allons expliquer certains concepts, afin de mieux comprendre ce pasteur et son groupe fondamentaliste et évangélique.

\section{Définition de quelques concepts}

\section{1 Évangélicalisme}

L'évangélicalisme a gardé les principes du protestantisme tels que la responsabilité individuelle, mais il insiste sur le salut personnel et a remplacé le baptême des enfants par celui des croyants adultes. Le protestantisme évangélique peut se définir à partir des principes de base de la Réforme protestante: Sola Gratia, Sola Fide, Sola Scriptura, Ecclesia Reformata, Semper Reformanda Est. Quatre critères le décrivent: la conversion, le biblicisme, l'activisme et le crucicentrisme (Bebbington 1989, 2-3). L'évangélicalisme est un "mouvement» qui se scinde en un nombre incommensurable de mouvances, partant du libéralisme au conservatisme (Rigal-Cellard 2003, 11). Rejetant toute religion établie, l'évangélicalisme n'est pas structuré de façon hiérarchique et se compose d'individus et de congrégations ${ }^{4}$. Enfin, on compterait environ $30 \%$ d'évangéliques aux États-Unis ${ }^{5}$.

3. Un seul décidait de ne plus supporter le CUFI qui était trop politisé et œcuménique à son gout.

4. L'évangélicalisme se définit également par l'existence de nombreuses rivalités entre les diverses dénominations qui le composent. Il existe beaucoup de confessions, de dénominations, de points de vue théologiques différents entre les grandes traditions (mainline church) et les Églises dites indépendantes dans l'ensemble du paysage états-unien. Il est donc impossible de généraliser l'étude d'une seule assemblée évangélique à tout le mouvement.

5. D'après les estimations du sondage «US-Evangelicals How Many Walk the Walk? ", fait en mai 2005 par le centre Gallup, on dénombre environ $22 \%$ d'évangéliques aux États-Unis qui répondent aux critères stricts du cœur de l'évangélicalisme, soit un Américain sur cinq (Winseman 2005). Or, selon une autre étude du Gallup de décembre 2005, nous pouvons considérer qu'entre 30 et $40 \%$ de la population étatsunienne est évangélique, ce qui représente une très large proportion (Newport et Carroll 2005). Il est donc difficile de dénombrer ces croyants, car tous les spécialistes ne s'accordent pas sur ces chiffres. 


\subsection{Fondamentalisme}

Le fondamentalisme américain (voir Ben Barka 1998), lui, s’inscrit dans un débat théologique interne au monde protestant évangélique, lequel opposait les modernistes aux fondamentalistes. Ce fondamentalisme, qui ne reconnaît aucune frontière entre le privé et le public, dérive de la publication intitulée The Fundamentals: A Testimony to the Truth. À partir de 1876, des baptistes accompagnés de presbytériens se rassemblèrent annuellement afin de programmer un retour aux «fondamentaux ", c'est-à-dire aux "valeurs authentiques ", selon eux, du christianisme. Cherchant à influencer le domaine public, ils se replièrent rapidement sur eux-mêmes après le "Procès du Singe ${ }^{6}$ ", car les médias et les libéraux ridiculisèrent ces chrétiens, considérés comme des arriérés. Ils créèrent alors leurs instituts bibliques et leurs écoles, tout en développant une sous-culture. Même si ce mouvement semble antimoderne, les fondamentalistes font maintenant largement usage de moyens technologiques, comme les médias sociaux. Les évangéliques du cercle de Hagee préfèrent le terme "chrétiens" ou l'expression "chrétiens bibliques»(Bible-believing Christian), et ne se revendiquent pas ou très rarement comme des fondamentalistes.

\subsection{Mouvement pentecôtiste ou charismatique}

Hagee appartient aussi au milieu dit charismatique, lui-même issu des milieux pentecôtistes. Ces cercles représentent la tendance la plus émotionnelle de l'évangélicalisme et se focalisent sur le baptême du Saint-Esprit: les pentecôtistes pensent vivre ce que les apôtres auraient expérimenté lors de la Pentecôte, d'où leur nom. Ils parleraient des langues incompréhensibles ou étrangères. La glossolalie est le nom scientifique du "parler en langues »: le croyant parlerait la langue des anges en prononçant des syllabes incompréhensibles, que seul Dieu comprendrait ainsi que certains croyants. D'autres pratiques curieuses accompagnent ce phénomène: le don de prophétie (1 Co 14,3-4) qui permettrait au croyant de révéler l'avenir, le don de délivrance ou d'exorcisme, le don d'interprétation des langues, le don de guérison (Ac 20,8-12) et le don «des paroles de connais-

6. Cette affaire, appelée aussi le procès de Scopes, se passa à Dayton dans le Tennessee et opposa les chrétiens conservateurs et créationnistes aux libéraux. Le professeur d'une école publique, John Thomas Scopes, fut condamné à verser une amende de cent dollars, car il avait enseigné la théorie évolutionniste à ses élèves. Il avait enfreint la loi de l'État du Tennessee dite le Butler Act qui interdisait aux enseignants de réfuter le créationnisme. 
sance» (1 Co 10,1-6). Notons que le terme «charismatiques» qualifie les évangéliques qui pratiquent ces dons et qui ne sont pas membres des assemblées pentecôtistes telles que les dénominations appelées Church of God in Christ ou les Assemblées de Dieu (Balmer 1999, 114-134). Les croyants qui vont dans des assemblées pentecôtistes sont appelés des pentecôtistes et les membres de Cornerstone sont des charismatiques ${ }^{7}$.

\subsection{Sionisme chrétien}

Le mouvement sioniste évangélique auquel Hagee appartient se fonde sur cinq thèmes majeurs: (1) le rejet de la théologie de la substitution (cette théologie dit que l’Église a remplacé Israël dans le plan de Dieu et les prophéties pour Israël dans l'Ancien Testament sont attribuées à l'Église); (2) la théologie de l'injonction (cette théologie est fondée sur la croyance en Genèse 12,3 qui demande de bénir Israël et sur Ésaie 62,1 : défendre Israël publiquement $^{8}$; (3) la théologie de la prophétie (les évangéliques du cercle hagien croient que les prophéties de l'Ancien Testament ne sont pas caduques et que la Bible aurait prédit la restauration d'Israël (1948) ainsi que la capture de Jérusalem (1967) par les juifs) avant le retour du Christ; (4) l'importance de l'histoire (ne pas reproduire les erreurs du passé, prévenir tout Holocauste, interpréter l'histoire à travers le prisme biblique). Enfin, un dernier thème représente ce mouvement: (5) l'argumentation sioniste, laquelle soutient Israël en tant qu'État historique qui a le droit de se défendre contre des attaques terroristes, et veut le retour des juifs en Israël, etc. (voir Batut-Lucas 2014).

Rétrospectivement, le sionisme évangélique puise ses racines en Grande-Bretagne et dans le restaurationisme ${ }^{9}$ qui s'est développé avec le protestantisme. Certains croyants pensaient que les juifs se convertiraient au christianisme et retourneraient dans leur terre ancestrale avant le retour imminent de Jésus. La London Jews Society cherchait ainsi à convertir les juifs (Cohn-Sherbok 2006, 21-22; Sizer 2006, 27-28). En 1891, l'évangélique William Blackstone, le père du sionisme évangélique états-unien, publiait une pétition, le "Mémorial de Blackstone» (Merkley 1998,

7. Discussion avec les membres de Cornerstone Church, mai 2013.

8. Dans la version de la Bible Louis Second (1910) qui se rapproche plus des versions utilisées par le groupe de Hagee, Gn 12,3 dit: «Je bénirai ceux qui te béniront, et je maudirai ceux qui te maudiront »; Is 62,1 dit : «Pour l'amour de Sion, je ne me tairai point».

9. Croyance dans la restauration des juifs au sein de leur terre ancestrale et biblique. 
59-68), laquelle soutenait politiquement le retour des juifs en Palestine. Plus récemment, dans les années 1970, la Droite chrétienne a pris son essor: un de ses piliers a été la défense d'Israël (voir Ben Barka 2006; Belin 2011; Fath 2005; Perani 2004). Ce mouvement est donc passé de la croyance à la politique. Le discours défendant Israël et les juifs a de plus en plus évacué les croyances eschatologiques au profit d'arguments philosémites, injonctifs et idéologiques.

Ainsi, ces notions permettent de mieux comprendre ce que croit Hagee et dans quel milieu il a évolué. Cela dit, il a voulu que le CUFI soit chrétien pro-Israël au sens le plus large possible.

\section{La formation de John Hagee}

\subsection{De l'enfance à l'âge adulte}

John Charles Hagee est né le 12 avril 1940 à Channelview, au Texas. Fils du révérend évangélique William Bythel Hagee et d'une mère nommée Vada, il est le deuxième de quatre garçons. Enfant chétif et malade, il grandit dans une famille modeste très pratiquante qui prêchait l'Évangile à travers la musique (Hagee 2007, 9). De plus, son enfance fut marquée par Israël:

For fifty years, my father was a minister of the gospel and a Bible Scholar.

Many evenings our family gathered around the dining table as my father taught us from the Bible that the Jewish people were "the apple of God's eye.» (Hagee et Ketterling 2008, 9)

Son enfance fut également bercée par la théologie du prémillénarisme dispensationaliste qu'il enseigne régulièrement à ses ouailles. Premièrement, le prémillénarisme de Hagee dit que le Christ viendrait enlever surnaturellement les "vrais» chrétiens (l'Enlèvement ou la seconde venue de Jésus) avant l'Armageddon ${ }^{10}$, la bataille finale entre les forces du Mal - ceux qui ont décidé de suivre Satan et qui ne sont pas des chrétiens "régénérés " — et les forces du Bien - les élus et le Christ; puis ensemble ils règneraient sur terre pendant le Millenium, une période de 1000 ans. Ils seraient enlevés en laissant "derrière eux" l'humanité, juste avant les tribulations, lesquelles dureraient sept ans. Deuxièmement, le dispensationalisme est un courant théologique propagé par John Nelson Darby (1800-1882). Darby

10. Le mot "Armageddon" est dérivé de Megiddo, nom d'une plaine en Israël où la bataille finale doit se dérouler, selon le livre de l'Apocalypse. 
scinda l'histoire de l'humanité en sept dispensations, dans lesquelles Dieu aurait un plan différent pour les hommes. Chaque étape se termine par l'échec de l'homme qui ne parvient pas à accomplir la volonté divine. Après le temps de l'Innocence (Éden) viennent ceux du Déluge, de la Gouvernance humaine (Babel), laquelle est suivie de la période de la Promesse avec Abraham, de la Loi sous Moïse, du temps de la Grâce (l’Église). Enfin survient le Royaume terrestre de Christ (Millénium). Darby enseignait qu'Israël serait restauré, que les prophéties au sujet d'Israël dans la Bible n'étaient pas caduques et que l'Église n'avait pas remplacé Israël.

Hagee fut ainsi marqué par les prédictions dispensationalistes de son père au sujet de la restauration d'Israël, ce qui forgea sa passion pour Israël:

It was the first time I ever saw tears in my father's eyes. Of course, I don't remember the exact words spoken by the announcer, but the news report would have been taken from this telegram, a copy of which is in the presidential Library of Harry Truman [...] When the announcer said, "the new state of Israel, " my father's eyes filled with tears. I knew something momentous had just happened. (Hagee 2007, 10)

Il développa en parallèle un philosémitisme, puisque son père lui enseignait également l'amour pour le peuple juif: "The only person that I have heard teach the love of God for the Jewish people was my father » (Entretien avec John Hagee 2011).

Plus tard, il devint à son tour pasteur en reprenant le flambeau d'une grande lignée pastorale représentant ainsi la cinquième génération de sa famille à exercer ce ministère. L'ancêtre de Hagee qui immigra en Amérique était morave, de la frontière suisse allemande (Hagee est en fait le quarante-septième descendant à vivre en Amérique) et il était en quête de liberté religieuse quelques années avant l'indépendance des colonies américaines.

Bien que ce soit un "merveilleux» prêcheur selon ses admirateurs, devenir un prédicateur était la dernière chose que John Hagee avait en tête pendant son adolescence. Rebelle à la religion, seul le sport comptait pour lui. Toute sa jeunesse, il vénérait le football américain au lieu d'adorer Dieu. Toutefois, il était un excellent élève de John H. Reagan High School, à Houston. Alors qu'il faisait de la trigonométrie un dimanche matin de l'année 1958, assis au fond de l'église de son père, il aurait eu une expérience spirituelle et aurait répondu à l'appel de conversion évangélique (Hagee et Ketterling 2008 citent Douglas, 5 et 7). Comme la plupart des convertis évangéliques, il changea radicalement de vie. Il décida de quitter 
son lycée pour un Institut biblique, la Southern Bible Institute, qui deviendra la Southwestern Assemblies of God University. Hagee fit des études de premier cycle à l'aide d'une bourse de football américain et devint membre de la liste d'honneur du Doyen. Il reçut une formation théologique à la Southwestern Bible College où il chanta dans la chorale Harvester. Il obtint par la suite un master à North Texas State University, puis un doctorat honorifique à l'Oral Roberts University. Il reçut également deux doctorats honoris causa, l'un du Canada Christian College à Toronto au Canada, l'autre du Netanya Academic College en Israël.

Sur le chemin d'une destinée religieuse qui semblait déjà tracée, il prêcha pour la première fois à l'âge de dix-sept ans, mais il débuta dans la pauvreté avant de bâtir son empire religieux et d'en vivre plus qu'aisément: il eut un deuxième emploi pour subvenir à ses besoins. Pendant un an, il dormit dans un garage tandis qu'il commençait à peine à prêcher. Il partagea cet espace minuscule avec un grand chien. Il devint vite célèbre pour sa façon de prêcher avec son accent texan nasillard, à la fois directe et simple. Son attitude positive face à l'adversité compléta sa renommée de prédicateur. Pour lui, rien n'était impossible (Hagee et Ketterling 2008 citent Douglas, 5, 7 et 85). Doctrinalement, Hagee s'inspire aussi des enseignements du prédicateur évangélique britannique Dereck Prince (19152003), notamment ceux sur Israël (Entretien avec John Hagee 2011) dont un bon nombre sont classés et rangés dans la bibliothèque de son bureau personnel du campus religieux, lieu où nous l'avons interrogé.

\subsection{Vers un empire religieux}

Après avoir servi en tant qu'évangéliste, Hagee dirigea en 1966 une nouvelle assemblée baptisée Trinity Church sur Nacogdoches Road à San Antonio au Texas. Au début de son ministère, John Hagee était à la fois pasteur et chef du département musical. L'Église de Castle Hills fut la deuxième congrégation dans laquelle il prêcha de 1975 à 1987. En mai 1975, un nouveau bâtiment fut construit pour accueillir mille six cents personnes assises. Celui-ci fut rapidement comble, obligeant Hagee et son équipe à passer d'un à trois services religieux pour recevoir tous les participants. Trois mille membres furent ainsi accueillis alors que Hagee avait, à ses débuts, dirigé une assemblée de seulement vingt-cinq fidèles. Une autre église se construisit encore: il s'agit de l'actuel bâtiment de Cornerstone Church, "Église de la pierre angulaire » en français, qui ouvrit ses portes le 2 août 1987. Cornerstone contient cinq mille places et se situe 
non loin d'une sortie d'autoroute de San Antonio, la Loop 1604. Sur le bord de l'autoroute, un énorme totem publicitaire, comme ceux des restaurants McDonald's, indique l'horaire des services religieux, les activités et le nom des prédicateurs.

Selon les membres, bien que beaucoup de personnes aient critiqué le nouvel emplacement de la megachurch, car il était à l'époque situé dans un lieu désertique, l'Église a grandi et, aujourd'hui, cette partie de la ville de San Antonio est très active et très peuplée. Pas de doute pour les membres, John Hagee était déjà un visionnaire et c'est aussi de cette façon qu'il devint de plus en plus populaire: s'il devait mener d'autres grands projets, il était devenu, à leurs yeux, crédible. Les fidèles pouvaient ainsi le suivre, car ce que Hagee entreprenait prospérait. Il influence ses disciples par son parcours.

Il n'y a rien de plus évangélique, pour certains pasteurs américains, que de posséder une gigantesque congrégation. Cela prouverait notamment le succès des messages de Hagee et le fait qu'il soit béni. Pour pouvoir administrer de telles masses de fidèles, le révérend Wallie A. Criswell (19092002) lui donna, de son vivant, des conseils pour diriger une megachurch. Criswell fut très engagé pour l'État hébreu, notamment avec la Droite chrétienne: "Dr Criswell was a dear friend and in my earlier pastoral career gave me a wealth of information on how to pastor a large church. He was a devout Zionist and we discussed the issue of Israel, often.» (Entretien avec John Hagee 2011).

Hagee devint le président de John Hagee Ministries (JHM). Cette "entreprise» religieuse diffuse ses messages par la radio nationale et la télévision à travers les États-Unis, l'Amérique entière, ainsi que dans deux cent trente-cinq nations du monde. Son campus possède deux énormes antennes paraboliques. Son empire religieux dispose de bureaux aux ÉtatsUnis (à San Antonio) et à l'étranger (à Toronto, au Canada et à Swindon, en Grande-Bretagne). Dans les années 1980, John Hagee obtint deux licences pour créer deux stations de télévision. Pour ce faire, il hypothéqua sa maison. Il décida d'accomplir un de ses projets, celui de prêcher dans le monde entier. C'est ainsi que Global Evangelism naquit dans une toute petite pièce avec une seule secrétaire. Poursuivant l'appel de la Grande Commission, il prêcha également à Bogota, en Colombie (Hagee et Ketterling 2008, 82). Il prêche régulièrement au Canada, au Kenya et en Angleterre.

Enfin, en 1975, il divorça de sa première épouse pour épouser Diana Castro avec qui il eut trois enfants. Il était déjà pasteur et père de deux 
enfants, issus du premier mariage - de ses deux mariages, il totalise treize petits-enfants ${ }^{11}$. Malgré le divorce, le pasteur, repenti et pardonné, a conservé sa popularité au sein de son groupe. Selon les membres et certains évangéliques que nous avons rencontrés, beaucoup qui ne résident pas à San Antonio souhaiteraient qu'il soit leur pasteur et qu'il vienne prêcher dans leur congrégation. Homme de vision qui prend des risques, formé et inspiré par des leaders évangéliques, il a su influencer beaucoup d'évangéliques.

\section{Hagee et Israël: du pasteur au dirigeant défenseur d'Israël}

\subsection{Sa production littéraire}

En plus d'être pasteur, John Hagee est l'auteur de plus d'une trentaine de livres dont quatre figurent dans la liste des meilleures ventes selon le New York Times. Même si les domaines prosélytes et mystiques, comme la guérison dans The Power to Heal (1991) ou l'exorcisme dans Invasion of Demons (1973) ont également été ses préoccupations, c'est au fur et à mesure des années qu'il a décidé d'écrire davantage sur Israël. Dans ce livre de 1973, Hagee parle déjà de la promesse divine aux gouvernements et à ceux qui bénissent ou maudissent Israël, mais il se limite essentiellement à l'antisémitisme, lequel est présenté comme provenant du camp des méchants dominés par le Diable (Hagee 1973, 93-94). En 1987, il publie son premier livre Should Christians Support Israel? consacré à la défense d'Israël, qu'il croit être biblique. Puis, sa production se politise de plus en plus dans les années 1990 en passant par des thématiques millénaristes et dispensationalistes: elle devient plus engagée envers l'État hébreu. Il publie Bible Positions on Political Issues (1992) dans lequel il s'oppose à l'avortement qu'il compare à un holocauste, au mouvement féministe et au mouvement écologiste.

11. Discussions avec les fidèles à l'occasion de notre dernière enquête de terrain de 2013. Il aurait trompé sa femme avec Diana et/ou ce serait sa femme qui aurait mal agi. Bien que nombre de fidèles en aient entendu parler, cette rumeur est souvent, parmi eux, démentie. Ils disent qu'ils n'en savent rien ou bien que c'est sa femme qui causait des problèmes dans leur couple. Bref, personne ne pouvait nous renseigner à ce sujet précisément, soit par ignorance, soit pour protéger leur pasteur et ne pas le juger. Ses deux dernières filles, Tina Ketterling et Sandy Hagee Parker, ainsi que Matthew Hagee chantent à l'église et sont reconnus pour leur voix. Matthew prend la relève à Cornerstone Church alors que le père s'occupe bien plus du CUFI. Les fidèles disent que Matthew, qui a une belle voix, a une "voix d'ange». Issus de son premier mariage, la fille ainée de Hagee travaille pour JHM et son premier fils dirige l'hôtel, Homewood Suites by Hilton, en face de l'église. 
Dans la lignée des discours de la Droite chrétienne et de nombreux fondamentalistes, il intègre ainsi des thèmes sur la politique intérieure et cherche à remettre l'Amérique dans le droit chemin, comme c'est le cas dans Day of Deception (1998). Il continue de publier sur l'eschatologie et Israël avec Beginning of the End (1996) et Final Dawn over Jerusalem (1997). Évolution notable, dans God's Candidate for America (2000), Hagee n'hésite pas à faire de ses lecteurs des value-voters pour les mobiliser dans la sphère publique. Dans ce but, il développe l'argument selon lequel il importe de traduire les idées religieuses en arguments séculiers afin de mieux influencer la société (Hagee 2000, 22), à l'instar de la Droite chrétienne des années 1970 et 1990 . Après le 11 Septembre 2001, Hagee se focalise sur la fin des temps, le terrorisme et la défense de l'État hébreu avec son best-seller, Attack On America (2001). Dans Bible Positions on Political Issues, qu'il a coécrit avec sa fille Sandy Hagee Parker, Hagee invite son lecteur à influencer l'opinion publique en s'engageant en politique au niveau local (Hagee et Parker Hagee 2009, 96). Puis, il publie en 2007 In Defense Of Israel, qui demeure le livre le plus emblématique sur l'apologie du sionisme chrétien évangélique et américain. Hagee veut ainsi influencer ses ouailles et ses lecteurs depuis des années à s'engager politiquement au nom de leurs convictions religieuses.

\subsection{Hagee et l'État d'Israël}

Le premier voyage de Hagee en Israël aurait été décisif dans son engagement vis-à-vis de l'État hébreu. En 1978, ce voyage religieux et touristique leur aurait permis, à lui et à sa femme, de rentrer "sionistes» en Amérique. Il dit y être tombé amoureux du peuple israélien et de sa nation: «But it was not until I took my first trip to Israel some thirty years later that my interest in Israel blossomed into an abiding love of the nation and its people. In 1978 Diana and I went on a ten-day trip to Israel with our first tour group. We went as tourists and came home as Zionists.» (Hagee 2007, 12).

Pour lui, cette nation a d'abord été une destination touristique, un pèlerinage fait de visites, avant de devenir sa patrie spirituelle. Il s'y est senti chez lui. En fait, il s'est identifié personnellement à cette nation, croyant qu'il y vivrait pendant le Millénium et que les racines de sa foi étaient connectées à la terre israélienne. Jérusalem est devenue sa "capitale» spirituelle, mais aussi mondiale, terrestre: "I experienced Israel as my spiritual home. For the first time, I fully understood that my spiritual roots were in this place. I have never felt about any other place on the earth as I felt about the city of Jerusalem.» (Hagee 2007, 12). 
C'est lors de ce voyage qu'il aurait eu une expérience surnaturelle en braquant ses yeux sur un rabbin qui priait contre le mur des Lamentations. Il narre avoir observé un homme vêtu de son châle de prière, qui priait à côté de lui en se courbant et en embrassant son livre de prières. Il comprit alors qu'il était trop ignorant au sujet de ses racines juives en tant que chrétien et se sentit inspiré par Dieu pour rassembler les juifs et les chrétiens dans un milieu public afin de partager le respect et l'amour:

When I went to Jerusalem for the first time in 1978, I was praying at the Western wall. It was there that I felt directed to do whatever I could to bring Christians and Jews together. I looked at my left and saw an orthodox Jew praying, rocking back and forth, covered with a praying shawl, wearing the yarmulke, tears on his face and knew this man loved God. I was aware I knew absolutely nothing about him [...]. And I felt the Lord speak to me and say: "I want you to bring Christians and Jews together in an arena where they feel mutual love and respect for each other.» I left the Prayer Wall with absolutely no idea of what had happened. (Entretien avec John Hagee 2011)

Une fois retourné aux États-Unis, il décida d'étudier l'histoire de l'Ancien Israël ainsi que les théologies chrétiennes qui ont influencé les persécutions contre les juifs. Après ce voyage et des recherches théologiques et historiques, il se destina à "montrer l'amour de Dieu » pour son peuple juif en rassemblant les chrétiens célébrant Israël:

I returned to America and began three years of intense individual scholarship to discover Israel's ancient history including the Crusades, the Spanish Inquisition, and the Roman Catholic doctrines born in the speech of Chrysostom, who has been called in history the "Golden Mouth", whose sermons gave birth to the seed of hatred that produced the Holocaust. When I came to know the role that theology had played in the persecution of the Jews, I thought for a way to demonstrate the love of God to the Jewish people. (Entretien avec John Hagee 2011)

Il faut noter que, dans les années 1970, beaucoup d'évangéliques ont mis de plus en plus en avant leur amour pour les juifs et Israël, ainsi que leur position politique pour défendre l'État hébreu, au point que certains ont abandonné le prosélytisme envers les juifs (Ariel 2013; Batut-Lucas 2014). Certains, nous l'avons vu, ont mis de côté, au fur et à mesure, leurs croyances eschatologiques qui les poussaient à s'intéresser à ces derniers.

Enfin, lors d'un autre voyage, Hagee aurait été inspiré non pas par la vue d'un homme juif, mais par le défilé de manifestants israéliens unis pour défendre la même cause. Il comprit alors le besoin pour ses compatriotes 
évangéliques d'être à la fois actifs et animés d'une passion au sujet de leurs croyances sur Israël (Hagee 1996, 29).

\subsection{Night to honor Israel}

C'est ainsi qu'il décida de mettre sur pied une première initiative. En septembre 1981, John Hagee fonda le «concept» des «Nuits pour honorer Israël » en invitant des pasteurs et des rabbins, qui ne cessent, depuis cette année-là, de se produire annuellement dans son assemblée en octobre, et régulièrement, sur tout le territoire américain: cette première rencontre deviendra l'une des activités phares du CUFI. Il s'agit d'un hommage nonprosélyte à la nation d'Israël et aux juifs du monde entier afin de promouvoir la reconnaissance et la compréhension entre les chrétiens et les juifs. Contrairement aux médias qui dénonçaient le bombardement israélien d'un réacteur nucléaire iraquien, Hagee pensait qu'Israël avait sauvé le monde en ôtant toute chance d'armement nucléaire à Saddam Hussein. Il décida donc de remercier cordialement le gouvernement israélien en réunissant tous les chrétiens pour honorer Israël. Pour lui, cet État venait, en effet, de «faire une faveur au monde » :

That way was made manifest in 1981 when the Israel Defense Force bombed the nuclear reactor in Iraq and removed nuclear weapons from the hands of Saddam Hussein. The global and the American media were hateful towards Israel for what they had done. I felt Israel has done the world a favor and that we, as Christians, should express our gratitude by having a city-wide «Night To Honour Israel». The first «Night To Honour Israel » was set in September 1981. (Entretien avec John Hagee 2011)

En fait, Hagee pense qu'Israël est l'allié des États-Unis, la seule démocratie au Proche-Orient alors que l'Iran serait l'ennemi numéro 1 des Israéliens et des Américains et que les pays arabes et musulmans seraient trop instables. Sa vision de l'islam est négative, craignant l'émergence d'un califat mondial. Pour lui, défendre Israël des terroristes et de ses ennemis, c'est défendre sa nation en retour. Selon sa perspective dualiste, il croit que les États-Unis et l'État hébreu seraient dans le camp du Bien et que tous ceux qui ne soutiendraient pas Israël appartiendraient au camp du Mal. Le monde serait alors plongé dans un combat cosmique dans lequel Israël serait au cœur.

Ce concept des soirées honorant Israël, qui provoqua de vives controverses, voulait montrer un soutien massif pro-Israël à l'encontre des critiques envers Israël. Après avoir reçu des menaces de mort, Hagee décida 
pourtant de maintenir sa célébration. Lors du service honorant Israël à l'Assemblée de Trinity, et comme nous l'ont narré quelques croyants, un homme entra et s'approcha de la scène, l'arme pointée en direction du pasteur, et lui demanda de s'agenouiller. L'homme tira, mais aucune balle n'atteignit le pasteur. Le tireur fut aussitôt plaqué au sol par les fidèles puis arrêté par la police. Cet épisode fut ensuite rapporté dans la presse locale. Pour les croyants, il s'agissait d'un miracle. John Hagee aurait été choisi par Dieu pour défendre Israël. Pour le pasteur, Dieu aurait été de son côté puisqu'il bénissait Israël. Dieu lui aurait ainsi sauvé la vie (Hagee et Ketterling 2008, 10). Désormais, rien ne pouvait plus stopper l'ambition de Hagee afin de prouver au Diable et aux personnes qui ne défendent pas Israël qu'ils avaient tort de lutter contre les politiques divines.

Pour ce premier rassemblement, un seul pasteur baptiste, le Dr Fanning, répondit positivement, alors que cent cinquante pasteurs en tout avaient reçu l'invitation. Un rabbin avec lequel Hagee sympathisa, Aryeh Scheinberg, accepta d'y venir (Hagee 2007, 36-43), et il semble qu'il ait assisté à tous les autres depuis. Cette célébration réussie deviendra le tremplin vers une organisation nationale.

L'action de Hagee souleva des polémiques tant du côté des évangéliques que du côté des juifs. Les premiers enseignements pro-Israël de Hagee ne furent pas toujours bien perçus par les évangéliques qui, à cette époque, ne comprenaient pas pourquoi il ne cherchait pas à convertir les juifs, au lieu de perdre son temps à leur exprimer son amour. En fait, son objectif n'a jamais été prosélyte:

When I began teaching Christians should be pro-Israel that day, both Christians and Jews thought I was wrong. The reason Christians were upset was that I was having massive rallies expressing the love of God to the Jewish people and they thought I should be trying to win them to join our church. It was not my objective. (Entretien avec John Hagee 2011)

De plus, le groupe de Hagee s'est attiré les foudres des partisans de la paix au Proche-Orient, comme les médias, beaucoup de musulmans, des juifs ${ }^{12}$ et des chrétiens de diverses confessions. Le désir du groupe serait de hâter le retour du Christ au nom d'un philosémitisme et d'une lecture litté-

12. Certains juifs croient que ces évangéliques ne les aiment pas pour ce qu'ils sont, mais pour le rôle attribué dans leur eschatologie. Ils représenteraient plus des instruments au service de la fin des temps. Cet amour passerait finalement pour un antisémitisme latent, puisque ces évangéliques leur prédisent un avenir très sombre: à la fin des temps ils devraient se convertir au christianisme ou périr. 
rale de la Bible. Hagee et ses disciples, eux, se défendent toujours d'être des antisémites camouflés et réfutent l'accusation d'avoir des intentions non déclarées: celles d'amplifier le conflit au Proche-Orient afin d'accélérer la fin des temps. Les médias et les détracteurs du mouvement ont insisté sur leurs croyances eschatologiques pour condamner l'action de ces évangéliques ou pour écrire des articles sensationnels. Pourtant, certains juifs la relativisent: le bras droit de Hagee au CUFI, David Brog, ou le porte-parole Ari Morgenstern que nous avons interrogé, tous deux juifs, réfutent ce genre de réquisitoire au nom d'une cause commune: la défense de l'État hébreu au Proche-Orient, et d'un amour sincère pour Israël et les juifs. Hagee et ses disciples mettent en avant leur philosémitisme et leur action fondés sur l'obéissance stricto-littérale des Saintes Écritures. Notons qu'il ne faut pas confondre motivation et croyance. Hagee croit en la fin des temps mais ne souhaite pas la provoquer. Ce pasteur prêche également que seul le SaintEsprit ouvrirait les yeux des juifs pour se convertir au Christ en se fondant sur la conversion de saint Paul sur le chemin de Damas. Changement remarquable dans les convictions eschatologiques, ces chrétiens ne doivent pas convertir les juifs, et attendent plutôt un acte surnaturel de Dieu lui-même en leur faveur (Hagee 2007, 102, 150, 152 et 200).

\subsection{Christians United For Israel (CUFI)}

Après son écrit à succès Jerusalem Countdown ${ }^{13}$ (2006), Hagee passa enfin à l'institution chrétienne sioniste pour pouvoir fédérer le maximum de croyants. Hagee avait besoin d'une structure publique, politiquement crédible pour se faire «entendre» nationalement et influencer les hommes

13. En 2005, Hagee écrit Jerusalem Countdown, livre qu'il publia en 2006 quelques semaines avant de lancer officiellement le premier sommet du CUFI et qui devint rapidement un best-seller sur la liste de USA Today. Il y raconte que la Bible prédit une coalition Pan-arabo-russe qui attaquerait Israël, mais que cette nation serait miraculeusement sauvée par Dieu. La bataille de l'Armageddon menée par l'Antéchrist et notamment la Chine s'ensuivrait; ensuite le Christ-Roi reviendrait vaincre les forces du Mal pour bâtir son royaume sur terre. Dans ce livre, le pasteur déclare aussi avoir des contacts qualifiés et haut placés, dont un, anonyme, qui lui aurait expliqué que l'Iran serait rapidement doté de l'arme nucléaire. Sans vérifier ce fait par d'autres sources, il est convaincu que l'Iran commande le terrorisme islamiste et qu'il est nécessaire d'arrêter le plus vite possible le programme nucléaire du dirigeant iranien. Ce livre semblerait vouloir préparer les membres de son groupe à entrer dans la mouvance institutionnelle de 2006 qu'il était sur le point de lancer. Pour lui, toute négociation avec l'Iran ne servirait à rien puisque Ahmadinejad aurait cherché à envahir le monde avec l'islam et à rayer Israël de la carte (Hagee 2006, vii). En 2015, Hagee croit toujours que l'Iran souhaite attaquer Israël. 
politiques à prendre parti pour l'État hébreu. En février 2006, le pasteur Hagee fonda ainsi le CUFI. Cette association offre tous les moyens possibles aux associations para-ecclésiales et aux Églises de parler en faveur de la défense d'Israël.

D’une part, le CUFI veut: (1) éduquer les chrétiens au sujet des impératifs bibliques et moraux pour soutenir Israël; (2) communiquer les perspectives favorisant l'État d'Israël, sur le plan des politiques publiques. D'autre part, le CUFI met en exergue une devise afin de justifier littéralement et bibliquement son action publique en faveur d'Israël: «For Zion's sake, I will not keep silent» (Is 62,1).

Les thèmes des discours et des actions du CUFI demeurent constants. Le leitmotiv «Israël, tu n'es pas seul» est fréquemment repris dans les exposés qui portent sur: la menace nucléaire iranienne; la lutte antiterroriste; le Hezbollah, le Hamas et l'islam radical; la lutte contre l'antisémitisme; le "plus jamais ça» ou l'horreur de l'Holocauste de la Seconde Guerre mondiale. Enfin, depuis 2014, le CUFI compterait, selon le site internet www.cufi.org, environ 2 millions de membres et il est soutenu par le Premier ministre israélien Benyamin Netanyahou, qui est très proche du pasteur et qui intervient régulièrement par visioconférence au sommet du CUFI qui a lieu en juillet annuellement. Le fait que leur pasteur soit ami avec Netanyahou est très valorisé par les membres.

En 2006, Hagee était tellement ambitieux qu'il osa qualifier son mouvement de "géant du sionisme chrétien ». Ce "géant» représente surtout le CUFI et pas forcément tous les chrétiens. On y trouve des juifs, des mormons, etc., et surtout des évangéliques (quoique tous les évangéliques ne soient pas sionistes ou n'acceptent pas les thèses de Hagee). Il utilise en fait des termes forts et médiatiquement parlants, gonflant le nombre d'adhérents au mouvement: "I want to say this as clearly and plainly as I possibly can: Israel, you are not alone. Ladies and Gentlemen, it's a new day in America. The sleeping giant of Christian Zionism has awakened. Fifty million Christians are standing up and applauding the State of Israel. » (Hagee $2007,2)$. Et ce, pour mieux influencer les sphères médiatiques et surtout les élus politiques, le groupe de Hagee représentant un vivier de voix potentielles. Enfin, Hagee incarne une personnalité charismatique.

\section{Une figure charismatique}

Outre ses talents de prédicateur et de dirigeant, Hagee se présente comme un chanteur et un musicien accompli, à la fois pianiste et saxophoniste. 
Son album de 2011, A Lifetime of Music, dans lequel il joue du saxophone, s'inspire de la musique de son enfance. Sa polyvalence et l'expression de ses divers talents, expression largement diffusée, contribuent à sa popularité dans le milieu évangélique.

Ses fidèles disent qu'il fait preuve d'une «foi de bulldog» et apprécient son style de prédication. Ses sermons sont ponctués de notes d'humour, précisons conservatrices, républicaines et fondamentalistes, qui font rire l'auditoire. Il fait flèche de tout bois. Pour les fidèles, il ne caresse pas dans le bon sens du poil les croyants, le péché ou bien les dérives de son pays. Son message est à prendre ou à laisser. Un fidèle raconte:

$\mathrm{He}$ is an old time Bible preacher. [...] He preaches straight out from the Word of God. I think he could be a watchman. I do not think he is a prophet because all this stuff comes from the Bible. [...] He is a kind of voice for the United States to tell, to warn what is going to happen. Although he gets his information from the Bible, I am sure he gets some by divine way. I think he is more a gospel-preaching preacher. No apologies. He does not apologize to anyone. He is telling what it is and that is it. No compromise. That is what he is. (Croyant 1 2011)

Homme de caractère et de charisme sur scène, c'est aussi un homme fortement apprécié pour sa convivialité. Les fidèles disent rêver de manger à sa table, car cela représente un honneur et certains ont déjà été invités à un souper, une expérience qu'ils aiment narrer. Ils déclarent également que leur pasteur est «oint ", "béni », "audacieux » et «très intelligent »: " $\mathrm{He}$ is anointed by God and blessed because he supports Israel. He is very smart. He knows a lot; he researches. He is very bold.» (Croyant 2 2011). Ces autres propos de fidèles expriment l'admiration et l'attachement:

$\mathrm{He}$ is a fireball that is compassionate with love. [...] He is so loving and caring about everyone in his congregation and outside. [...] But he wants us to share God's love and to teach others to love Israel, to love everyone and to pray for their salvation. He is not just there to teach. [...] He is amazing. He is a man of God. (Croyant 3 2011)

Et encore:

[...] I would describe him as the most courageous man in the pulpit, I believe, in the world. He is one of the few men that I know who, in the face of danger and ridicule, is unwavering. I have never met a pastor anywhere in the world, in the countries where I have been in, especially those I have watched on TV, I have never seen a pastor who has made such a bold stand for what he believes in his heart is the right thing to do. So I see him as a 
truth bearer, a kind of watchman in these last days. [... He] is more of a general in a big army, the army of God. (Croyant 4 2011)

Son charisme et sa maîtrise du discours le placent au rang d'homme politique et de vedette du milieu, ce qui crée un fossé entre les simples fidèles et ce prédicateur. Hagee est reconnu comme un leader hors du commun, élu de Dieu pour les croyants. Cela dit, il ne remplace pas Dieu, et comme le précisent des adeptes, c'est d'abord «un homme de Dieu » qui "aime le Seigneur ${ }^{14}$ » et sa congrégation. Les ouailles croient donc que Dieu utiliserait ce prédicateur comme un canal par lequel il passerait son message aux hommes.

Certains le comparent à un prophète même si Hagee ne se désigne jamais comme tel. Pour Hagee, ceux qui le prennent pour un tel homme ignorent le fait qu'il obéit fondamentalement aux Saintes Écritures. Il justifie son action comme simple obéissance biblique: «I am only obedient to the very Word of God. And some people think of me of a prophet because I am only obeying the Scriptures. They just don't know the Scriptures. » (Entretien avec John Hagee 2011).

Pour comprendre le leadership de Hagee, nous pouvons nous fonder sur des concepts classiques de la sociologie ${ }^{15}$. On trouve de cette manière chez Max Weber certains traits observables ou attribués à Hagee, qui l'associent en partie au prophète, à la figure charismatique ou au réformateur religieux ${ }^{16}$ : le charisme, pour l'aspect un peu autodidacte, la reconnaissance par la communauté, l'itinérance et la prédication émotionnelle.

Hagee possède en effet plusieurs caractéristiques du prophète protestant, tel que Weber le définit, même s'il ne s'autoproclame pas prophète - nous venons de le mentionner - et, qui plus est, demeure rémunéré pour ses prédications, ses livres et ses conférences. Toutefois, Hagee, qui croit être le porteur du message de Dieu, c'est-à-dire le message chrétien sioniste qui défend Israël et les juifs, proclame un commandement divin, celui de parler au nom d'Israël: «Par prophète, nous entendons ici un

14. Notre traduction.

15. Nous aurions pu nous pencher sur d'autres auteurs et des publications plus récentes, cela dit l'étude de Weber nous semblait pertinente ici.

16. Pour résumer rapidement le prophète de Weber: il s'agit d'un personnage, doué de charisme et autodidacte, qui s'autoproclame prophète et qui est sur le chemin de l'«itinérance » afin de prêcher sa doctrine divine en s'appuyant sur une révélation de Dieu sans demander aucun soutien familial, car il subvient à ses besoins lui-même et il rallie une communauté de croyants autour de son message, qui le reconnaissent pour sa vocation. Voir Weber (1995, tome 2). 
porteur de charismes purement personnels, qui en vertu de sa mission, proclame une doctrine religieuse ou un commandement divin» (Weber 1995, 190 , nous soulignons).

Selon les fidèles, Hagee leur dit que c'est sa "vocation" de rallier toute une communauté au nom d'Israël, ce qu'ils croient également: "[L]'élément décisif c'est la "vocation" personnelle. [...] Le prophète revendique son autorité en invoquant une révélation personnelle ou en se réclamant d'un charisme» (Weber 1995, 190).

Hagee ne prétend pas avoir reçu une révélation divine (si ce n'est quand il pense que Dieu lui aurait demandé de réunir les juifs et les chrétiens au mur des Lamentations), il dit avoir étudié l'histoire des persécutions juives, l'histoire de l'Église et surtout lu la Bible littéralement (Is 62,1 et $\mathrm{Gn} 12,3$ ); en ce sens, il répond à la définition de Weber qui distingue le prophète du réformateur. Weber dit que le réformateur n'a pas de «révélation nouvelle»(Weber 1995, 198), car il s'appuie littéralement sur la Bible.

Hagee persiste à répondre à l'appel divin pour Israël malgré les menaces de mort et d'attentat à la bombe, notamment pour les Nuits Pour Honorer Israël. Son courage et sa détermination suscitent l'admiration de ses fidèles:

The first time he did it in San Antonio, he had a bomb threat. Because of the bomb threat he realized, "I am going to do this again. " From a reputation perspective, you think this bomb would have stopped him. No, he has been under a bomb threat; the bomb has not stopped him. After so many years, nothing has stopped him. (Croyant 4 2011)

Sa communauté politico-religieuse le reconnaît comme chef des chrétiens américains défenseurs d'Israël:

Nous appellerons charisme la qualité extraordinaire [...] d'un personnage, qui est, pour ainsi dire, doué de forces ou de caractères surnaturels ou surhumains ou tout au moins en dehors de la vie quotidienne, inaccessible au commun des mortels; ou encore qui est considéré comme envoyé par Dieu ou comme un exemple, et en conséquence considéré comme un "chef» [Führer]. (Weber 1995, 320)

Hagee se démarquerait ainsi des autres pasteurs par son charisme et ses traits de caractère qui viennent de Dieu. Une croyante le compare à un chef, même si elle fait référence à un chef de cuisine de téléréalité, soit Gordon Ramsey qui est notamment connu pour son caractère autoritaire et qui aide les apprentis à exceller en cuisine comme lui; elle perçoit ainsi 
Hagee comme un modèle et un prophète qui l'aide à son tour à transmettre ses croyances :

I think he is a prophet. God has blessed him in many different ways. He has given him the gift of discernment and the gift to be able to speak. He has given him the gift of authority to go out there and to preach it to the people. [...] But when I came here, Pastor Hagee is the "Chef ", like Ramsey: you have to move to the next level, you have to be... God wants a warrior. God wants someone who is on fire. He has that gift to be able to bring people up to the next level. (Croyante 6 2011)

L'itinérance, selon Weber, est une autre caractéristique du prophète (Weber 1995, 191). Remarquons que Hagee parcourt les églises (par analogie bien entendu), se rend à Jérusalem et à Washington D.C. afin de transmettre son message aux États-Unis.

Souvent, les prédications de Hagee ont recours à l'émotion en suscitant la crainte de la malédiction pour bénir Israël, la tristesse en évoquant la Shoah, etc.: "[...] La véritable caractéristique des prophètes [est]: une véritable prédication émotionnelle [...]» (Weber 1995, 197).

Enfin, ses idées sur l'État d'Israël l'ont amené à bâtir sa propre communauté de sympathisants - le CUFI — qui s'approprie son message. C'est donc pour toutes ces caractéristiques que le pasteur John Hagee possède certains traits d'une figure charismatique, réformatrice et prophétique selon les perspectives wébériennes.

\section{Conclusion}

Outre ce sens sociologique, Hagee proclame souvent des oracles de malheur ou des jugements divins à venir si les chrétiens ou les États-Unis ne se conformaient pas à la volonté surnaturelle, notamment pour Israël, et n'éradiquaient pas le péché. Ce pasteur semblerait imiter les prophètes de la Bible en annonçant la sentence et la malédiction à ses auditeurs, en disant par exemple que la Louisiane aurait subi le jugement de Dieu pour expliquer les catastrophes climatiques, à cause de l'attitude de l'Amérique favorisant le retrait israélien de Gaza en 2005: «It started with George W. Bush when he told Israel to get your people out of the West Bank. On the same day they were relocated, a storm started and grew. It was called Katrina. Then, New Orleans was relocated.»(Gross 2008; Frederik 2008). John Hagee se fait porte-parole biblique, c'est-à-dire un médiateur de la volonté divine pour Israël sur terre. 
Malgré les embûches, Hagee est parvenu à son rêve: être le meneur d'une organisation rassemblant des chrétiens, surtout évangéliques, qui veulent défendre l'État d'Israël. Hagee aura réussi à marquer son temps, par le biais du CUFI et de sa médiatisation en criant son soutien à l'État hébreu sur tous les toits. Enfin, pour un croyant, son pasteur représente une sorte de prophète, tel Moïse et sa célèbre phrase qui insuffla l'idée de liberté: "laisse mon peuple aller». Pour Hagee, cette citation s'est transformée en «laisse mon peuple vivre! » et «laissez-lui sa terre»:

[Hagee] is like Moses who was in the desert. He was hanging out and now the time of the burning bush has been turned. [He] is delivering a message: let my people live. When we had Moses coming the first time with Pharaoh, it was «Let my people go ». Now it is «Let my people live». So I am just changing one word. Let my people live. Israel has already given their land. Let them live! What are we doing? Let their land stay. (Croyant 5 2011)

\section{Références}

Ariel, Y. (2011), «Messianic Hopes and Middle East Politics. The Influence of Millennial Faith on American Middle East Policies ", dans Revue LISA/LISA, 9/1, p. 13, disponible sur <http://lisa.revues.org/4165> (consulté le 19 février 2012).

(2013), An Unusual Relationship. Evangelical Christians and Jews, New York, New York University Press.

Batut-Lucas, K. (2014), Le sionisme chrétien contemporain aux ÉtatsUnis, entre religion et politique. Thèse de doctorat, Faculté de Théologie et Sciences des Religions, Université de Montréal.

Balmer, R. (1999), Blessed Assurance. A History of Evangelicalism in America, Boston, Beacon Press.

Bebbington, D. (1989), Evangelicalism in Modern Britain. A History from the 1730s to the 1980s London, Boston, Unwin Hyman.

Ben Barka, M. (1998), Les Nouveaux Rédempteurs. Le fondamentalisme protestant aux États-Unis, Paris, Labor et Fides.

(2006), La droite chrétienne américaine, Les évangéliques à la Maison-Blanche, Paris, Privat.

Belin, C. (2011), Jésus est juif en Amérique. Droite évangélique et lobbies chrétiens pro-Israël, Paris, Fayard.

Conn-Sherbok, D. (2006), The Politics of Apocalypse, Oxford, Oneworld. 
FATH, S. (2005), «Le poids géopolitique des évangéliques américains. Le cas d'Israël », dans Hérodote, 4/119, p. 25-40.

(2008), Dien XXL. La révolution des megachurches, Paris, Autrement.

Frederik, D. (2008), «McCain Backer John Hagee Tempers Hurricane Katrina Comments ", 26 avril, disponible sur <http://latimesblogs. latimes.com/washington/2008/04/mccain-backer-j.html> (consulté le 29 juillet 2012).

Gross, T. (2008), "Pastor John Hagee on Christian Zionism, Katrina ", Entretien de John Hagee, NPR, 18 mai, disponible sur <http://www. npr.org/templates/story/story.php? storyId $=90508742>$ (consulté le 29 juillet 2012).

Hagee, D. et Ketterling, T. (2008), Celebrating Fifty Years in Ministry. John Hagee 50 years 1958-2008, Nashville, Thomas Nelson Publishers.

Hagee, J. (1973), Invasion of Demons. The Battle Between God and Satan in Our Time, Old Tappan, Revell.

(1987), Should Christians Support Israel?, San Antonio, Dominion Publishers.

(1991), The Power to Heal, San Antonio, John Hagee Ministries. (2000) God's Candidate for America. Letting Your Light Shine in a Dark World, San Antonio, Global Evangelism Publishing.

(2001), Attack on America, New York, Jerusalem, And The Role Of Terrorism In The Last Days, Nashville, Thomas Nelson Publishers. (2006) Jerusalem Countdown, Lake Mary, Frontline.

(2007) In Defense of Israel. The Bible's Mandate for Supporting the Jewish State, Lake Mary, Frontline.

Hagee, J. et Parker Hagee, S. (2009) [2004], Bible Positions on Political Issues, John Hagee and Global Evangelism Television.

Harding, S. (2000), The Book of Jerry Falwell, Princeton, Princeton University Press.

Kiracofe, C. (2009), Dark Crusade. Christian Zionism and US Foreign Policy, London/New York, I. B. Tauris.

Merkley, P. (1998), The Politics of Christian Zionism 1891-1948, London/ Portland, Frank Cass.

Newport, F. et Carroll, J. (2005), "Another Look at Evangelicals in America Today ", Gallup News Service, 2 décembre, disponible sur $<$ http://www.gallup.com/poll/20242/Another-Look-EvangelicalsAmerica-Today.aspx> (consulté le 12 janvier 2012). 
Rigal-Cellard, B. (2003), «La rhétorique de "l'axe du mal”, Études, septembre, p. 153-162.

Sizer, S. (2006), Christian Zionism. Road-Map to Armageddon?, Leicester, Inter-Varsity Press.

Spector, S. (2009), Evangelicals and Israel. The Story of American Christian Zionism, New York, Oxford University Press.

Van Ingen, L. (2010), "John Hagee Draws Large Crowd to Convention Center», 22 avril, <http://lancasteronline.com/article/ local/251526_John-Hagee-draws-large-crowd-to-conventioncenter. html\#ixzz1rd78vjxH> (consulté le 10 mars 2011).

Wacker, G. (2014), America's Pastor. Billy Graham and the Shaping of a Nation, Harvard, Belknap Press.

Weber, M. (1995) [1921, allemand 1971], Économie et Sociétés, 1/ Les catégories de la sociologie; 2/ L'organisation et les puissances de la société dans leur rapport avec l'économie, sous la direction de J. Chavy et É. de Dampierre, Paris, Plon.

Weber, T. (2004), On the Road of Armageddon. How Evangelicals Became Israel's Best Friend, Grands Rapids, Baker Academy.

Winseman, L. (2005), "U.S. Evangelicals. How Many Walk the Walk? », Gallup, 31 mai, <http://www.gallup.com/poll/16519/US-EvangelicalsHow-Many-Walk-Walk.aspx> (consulté le 2 septembre 2011).

\section{Résumé}

Cet article porte sur John Hagee et son parcours pour devenir le dirigeant du groupe chrétien sioniste le plus grand et le plus médiatisé aux États-Unis et dans le monde, le Christians United For Israel (CUFI). Depuis 2006, ce pasteur s'est fait connaître du grand public à cause de son soutien inconditionnel à l'État hébreu. Comment Hagee en est-il arrivé à créer le plus grand groupe de pression chrétien sioniste qui existe de nos jours? Comment est-il devenu l'ami d'Israël et ce chef de file si remarqué? De quel milieu évangélique est-il issu? Cet article veut ainsi comprendre comment un pasteur évangélique est devenu une figure politique médiatisée et comment il a pu rallier bon nombre d'évangéliques à le suivre dans son activisme pro-Israël. Hagee est une figure charismatique. Notre méthodologie se fonde sur des études de terrain, des entretiens et des observations. 


\section{Abstract}

This paper focuses on John Hagee and his path to become the leader of the largest and most publicized Christian Zionist group in the United States and the world, Christians United For Israel (CUFI). Since 2006, this pastor has become renowned for his support to the Hebrew State. How did Hagee come to create the largest Christian Zionist lobby existing today? How did he become such a friend of Israel and so remarkable a leader? What is his background? This article looks deeper at how an Evangelical pastor became a political public figure capable of rallying large numbers of Evangelicals to follow him in his pro-Israel activism. Hagee is a charismatic leader. Our methodology is based on field studies, interviews and observations. 